\title{
Replicas of the Kondo peak due to electron-vibration interaction in molecular transport properties
}

\author{
P. Roura-Bas, ${ }^{1}$ L. Tosi,${ }^{2}$ and A. A. Aligia ${ }^{2}$ \\ ${ }^{1}$ Dpto de Física, Centro Atómico Constituyentes, Comisión Nacional de Energía Atómica, Buenos Aires, Argentina \\ ${ }^{2}$ Centro Atómico Bariloche and Instituto Balseiro, Comisión Nacional de Energía Atómica, 8400 Bariloche, Argentina
}

(Received 26 October 2015; published 24 March 2016)

\begin{abstract}
The low temperature properties of single level molecular quantum dots including both electron-electron and electron-vibration interactions, are theoretically investigated. The calculated differential conductance in the Kondo regime exhibits not only the zero bias anomaly but also side peaks located at bias voltages which coincide with multiples of the energy of vibronic mode $V \sim \hbar \Omega / e$. We obtain that the evolution with temperature of the two main satellite conductance peaks follows the corresponding one of the Kondo peak when $\hbar \Omega \gg k_{B} T_{K}, T_{K}$ being the Kondo temperature, in agreement with recent transport measurements in molecular junctions. However, we find that this is no longer valid when $\hbar \Omega$ is of the order of a few times $k_{B} T_{K}$.
\end{abstract}

DOI: 10.1103/PhysRevB.93.115139

\section{INTRODUCTION}

The Kondo effect, originally discovered in metals containing magnetic impurities [1,2], is also observed in transport measurements through semiconducting [3-9] and molecular [10-19] quantum dots (QDs) in which the QD acts as a single magnetic impurity. While the semiconducting QDs are characterized by the tunability of its parameters and have served as platforms to study the one- and two-channel [3,5,7,20], as well as the SU(4) Kondo effect [21], the molecular QDs (MQDs) have allowed researchers to investigate, among different phenomena, the underscreened Kondo effect for spin $S>1 / 2[14,15,18]$ and quantum phase transitions driven by stretching $[14,22]$ or gate voltage $[15,18]$. Remarkably, MQDs incorporate the effect of phonons. Molecular vibration signatures have been observed in conductance measurements through a variety of molecules, such as $\mathrm{H}_{2}$ [23] and $\mathrm{C}_{140}$ [24]. Furthermore, experiments performed in the Kondo regime have revealed the presence of satellite peaks at finite bias which emerge together with the zero-bias anomaly in the differential conductance as a consequence of the interplay between the electron-vibration interaction and the many-body Kondo state [14,25-29].

Recently, Rakhmilevitch et al. reported on transport measurements through a copper-phthalocyanine $(\mathrm{CuPc})$ molecule connected to two silver contacts in a break-junction setup [29]. Their work focuses on the evolution with temperature of the side peaks observed in the differential conductance. The authors find that the maximum conductance of the side peaks increases when the temperature is lowered following the same dependence as the zero-bias Kondo peak. Specifically (since CuPc possess a spin-1/2 [29,30]) the intensity of both the zero-bias anomaly and the satellite peaks can be fitted with the same empirical expression for the temperature dependence of the equilibrium conductance of a spin-1/2 impurity, $G(T)$, which follows closely results obtained using the numerical renormalization group

$$
G(T, V=0)=\frac{G_{s}}{\left[1+\left(2^{1 / s}-1\right)\left(T / T_{K}\right)^{2}\right]^{s}},
$$

where $s=0.22[3,31], G_{s}$ is the conductance at temperature $T=0$, and the Kondo temperature $T_{K}$ is the only adjustable parameter. Outstandingly, the fitted Kondo scale for the satellite peaks located at bias voltage $e V \sim \pm \hbar \Omega \sim \pm 21 \mathrm{meV}$, agrees with the one obtained for the Kondo peak $\left(21 \mathrm{~K}<T_{K}<\right.$ $25 \mathrm{~K})$. This is an interesting result for two main reasons: First, it is not evident that the empirical expression Eq. (1) that correctly gives the universal temperature dependence of the conductance without vibrational modes can be applied when the Kondo phenomena is assisted by phonons. More surprising is the fact that Eq. (1) still works out of equilibrium, for fitting the conductance at finite bias voltages of the order of $|V| \sim \hbar \Omega / e$. While the satellite peaks have been studied theoretically before $[32,33]$, their dependence on temperature has not been analyzed.

Motivated by the experiment of Rakhmilevitch et al., in this paper we investigate theoretically the low-temperature transport properties of a single level molecular quantum dot including both electron-electron and electron-vibration interactions. In agreement with the experimental results, we obtain that the conductance of the two main satellite peaks follows the same temperature evolution as the corresponding Kondo peak, which means that these peaks are also a manifestation of the Kondo effect, when $\hbar \Omega \gg k_{B} T_{K}$, which is in fact the regime of the experiment. On the other hand, the statement is no longer valid when both energy scales are similar, $\hbar \Omega \approx 3 k_{B} T_{K}$. For $\hbar \Omega \leqslant k_{B} T_{K}$, the satellite peaks merge with the Kondo peak [34].

\section{MODEL AND FORMALISM}

We model the MQD with the Anderson-Holstein Hamiltonian [32-38] in which a spin-1/2 doublet of energy $E_{d}$ is connected to two metallic reservoirs and also coupled to a phonon mode of frequency $\Omega$, through the electron-phonon interaction $\lambda$. The Hamiltonian is

$$
\begin{aligned}
H= & {\left[E_{d}+\lambda\left(a^{\dagger}+a\right)\right] n_{d}+U n_{d \uparrow} n_{d \downarrow}+\sum_{\nu k \sigma} \epsilon_{k}^{v} c_{\nu k \sigma}^{\dagger} c_{\nu k \sigma} } \\
& +\sum_{\nu k \sigma}\left(V_{k}^{v} d_{\sigma}^{\dagger} c_{\nu k \sigma}+\text { H.c. }\right)+\Omega a^{\dagger} a,
\end{aligned}
$$

where $n_{d}=\sum_{\sigma} n_{d \sigma}, n_{d \sigma}=d_{\sigma}^{\dagger} d_{\sigma}, d_{\sigma}^{\dagger}$ creates an electron with spin $\sigma$ at the relevant state of a molecule (or quantum dot), $a^{\dagger}$ creates the Holstein phonon mode, $c_{\nu k \sigma}^{\dagger}$ creates a conduction electron at the left $(v=L)$ or right $(v=R)$ lead, and $V_{k}^{v}$ 
describe the hopping elements between the leads and the molecular state. We take the limit of very large Coulomb repulsion $U \rightarrow \infty$.

We use the noncrossing approximation (NCA) in its nonequilibrium extension $[33,39]$. The out of equilibrium NCA approach has proved to be a very valuable technique for calculating the differential conductance through a variety of systems including two-level QD's and $\mathrm{C}_{60}$ molecules displaying a quantum phase transition [18,40,41], among others. Furthermore, it is specially suitable for describing satellite peaks away from the zero bias voltage [42-44] and captures the universal behavior in the equilibrium conductance given by Eq. (1) $[45,46]$. The application of the NCA to the model and its limitations for large $\lambda$ are described in detail in Ref. [33].

The current through the molecule is calculated using the exact expression $[39,47]$

$I(V)=\frac{4 \pi e}{\hbar} \frac{\Gamma_{L} \Gamma_{R}}{\Gamma_{L}+\Gamma_{R}} \int d \omega \rho(\omega)\left(f\left(\omega-\mu_{L}\right)-f\left(\omega-\mu_{R}\right)\right)$,

where $\Gamma_{v}=2 \pi \sum_{k}\left|V_{k}^{v}\right|^{2} \delta\left(\omega-\epsilon_{k}^{v}\right)$ (assumed independent of energy) is the coupling of the molecule to the lead $v, f(\omega)$ is the Fermi distribution, and the spectral function of the molecule is given by $\rho(\omega)$, which we calculate using the NCA. The right and left chemical potentials $\mu_{v}$ of the metallic contacts are proportional to the bias voltage. For simplicity we assume a symmetric voltage drop $\mu_{L}=-\mu_{R}=e V / 2$. The results are not affected by this assumption.

The reduced intensity of the zero-bias peak in the experiment suggests that there is a large asymmetry between the two tunneling couplings $\Gamma_{v}$. This is usually the case in MQDs. We choose $\Gamma_{R} \approx 50 \Gamma_{L}$ (similar values do not affect our conclusions). We note that for ratios of the couplings larger than 10 (i.e., highly asymmetric devices), the differential conductance $G=d I / d V$ at bias voltage $|V| \lesssim k_{B} T_{K} / e$ reproduces the equilibrium spectral density of the Kondo resonance [48].

While the value of the vibration frequency is well defined by the position of the satellite peaks in the conductance measurements $(\hbar \Omega \sim 21 \mathrm{meV})$, neither the value of the total coupling $\Gamma_{R}+\Gamma_{L}$ nor the energy position of the Kondo active molecular level $E_{d}$ are clearly determined. Therefore, we analyzed several values of both energies to confirm that our conclusions remain the same. The estimated value for the electron-vibration interaction $\lambda$ that corresponds to a breathing mode of the $\mathrm{CuPc}$ molecule is found from ab initio calculations to be near $\lambda_{0}=6 \mathrm{meV}$. Since the Kondo scale strongly depends on the electron-phonon coupling [33], in the present work we tested several values of $\lambda$, from 6 to $12 \mathrm{meV}$. We verified that our analysis regarding the ratio $k_{B} T_{K} / \hbar \Omega$, holds for the whole set of $\lambda$ 's studied.

\section{THE MAIN KONDO PEAK}

We start by a brief review of the temperature evolution of the Kondo peak in Fig. 1(a) and the scaling law of the zero-bias conductance with Eq. (1) in Fig. 1(b) in the absence of the electron-phonon interaction. The asymmetry in $G(V)$ is due to the asymmetric $\Gamma_{v}$ [48]. For the parameters of the

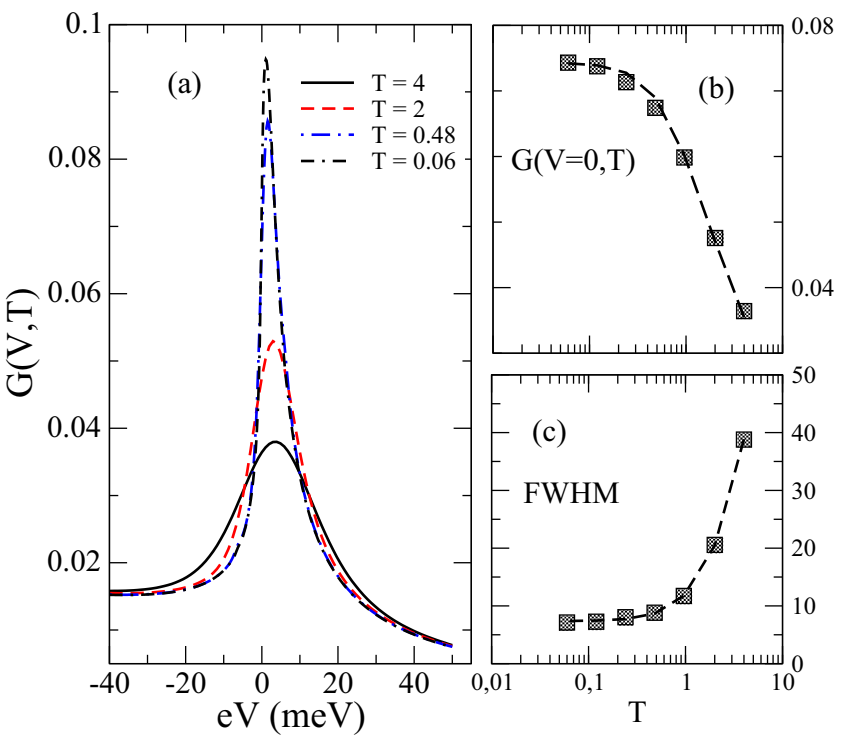

FIG. 1. (a) Differential conductance as a function of bias voltage for several temperatures $T$. Parameters in meV: $\Gamma_{R}+\Gamma_{L}=80, E_{d}=$ $-90, \lambda=0$. (b) Equilibrium conductance $G(V=0, T)$ as a function of temperature (squares) and the corresponding scaling using Eq. (1) (dashed line) being $T_{K}=43 \pm 1 \mathrm{~K}$. (c) FWHM of the zero bias anomaly as a function of temperature (squares) and the fitting function (see text) with $\alpha=9.5 \pm 0.5$ and $T_{K}=43 \pm 1 \mathrm{~K}$ (dashed line).

figure, the Kondo scale resulting from the fit using Eq. (1), $T_{K}=43 \pm 1 \mathrm{~K}$, remarkably agrees with the corresponding one in Fig. 1(c) extracted from a fitting of the full width at half maximum (FWHM) of the zero-bias anomaly using the expression $[49,50]$ FWHM $=\frac{1}{e} \sqrt{\left(\alpha k_{B} T\right)^{2}+\left(2 k_{B} T_{K}\right)^{2}}$, being in this case $T_{K}=43 \pm 1 \mathrm{~K}$, with $\alpha$ an extra fitting parameter. This expression for the FWHM gives a value of $2 k_{B} T_{K} / e$ at zero temperature and is expected to coincide with the FWHM of the equilibrium spectral density for large asymmetric devices in the Kondo limit [48]. We must warn the reader that the Kondo scale determined from the width of the spectral density can be $10 \%$ larger that the corresponding one obtained from the temperature dependence of the conductance [51]. The scaling of $G(V, T)$ for small $V$ and $T$ and also under an applied magnetic field has been investigated experimentally and theoretically $[7,8,16,45,52,53]$.

As we stated before, when the coupling to the vibration mode of the molecule is taken into account, it is not obvious that $G\left(T / T_{K}\right)$ and FWHM $(T)$ are still described with the same expressions. In Fig. 2(a) we show the differential conductance as a function of the bias voltage for several temperatures and $\lambda=12 \mathrm{meV}$. All the parameters are the same as in Fig. 1 . As the temperature decreases, the zero-bias peak emerges together with lateral satellite peaks at voltages corresponding to the energy of the vibration mode $e V= \pm \hbar \Omega= \pm 21 \mathrm{meV}$. Other satellites peaks with reduced intensity at multiples of the vibration energy should also be present [32,33], but they are beyond the scope of this paper. The intensity of the two main satellite conductance peaks increases with increasing $\lambda$ [32,33]. Figure 2(b) displays the zero-bias conductance as a function of temperature and the corresponding scaling result using Eq. (1). We obtained a Kondo temperature $T_{K}=20 \pm$ 


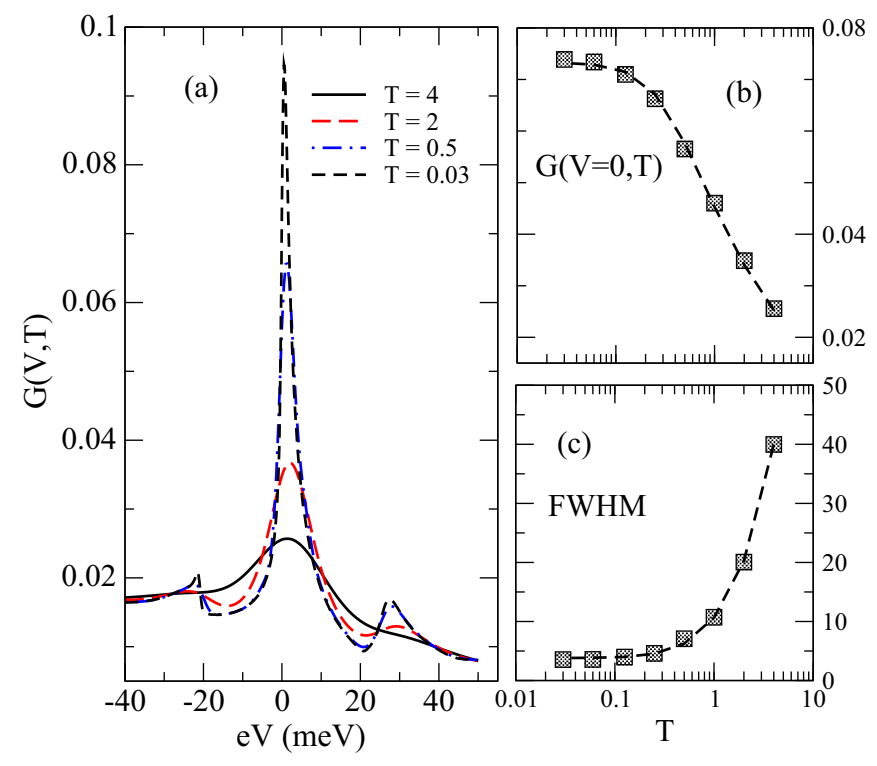

FIG. 2. (a) Differential conductance as a function of bias voltage for several temperatures and $\Omega=21 \mathrm{meV}$ and $\lambda=12 \mathrm{meV}$. Other parameters as in Fig. 1. (b) Equilibrium conductance $G(V=0, T)$ as a function of temperature (squares) and the scaling result using Eq. (1) (dashed line). (c) FWHM of the zero bias peak as a function of temperature (squares) and the fitting result (dashed line).

$1 \mathrm{~K}$, which is markedly reduced due to the effect of the electron-phonon coupling $\lambda$, but the decrease is smaller than expected from a Franck-Condon factor. The nontrivial reduction of $T_{K}$ with the electron-phonon coupling $\lambda$ has been discussed before $[33,34,38]$. We conclude that the only change in the temperature evolution of the zero-bias Kondo peak is given by the Kondo scale, but the universal behavior is not affected. The high precision of the fitting, with a correlation factor of 0.9995 , indicates that for low enough temperatures and energies $\left(k_{B} T \ll \hbar \Omega\right)$ the system behaves as a Fermi liquid with renormalized parameters in which the phonon mode is not active.

In Fig. 2(c) we show the FWHM of the zero bias anomaly as a function of temperature and the corresponding scaling being $\alpha=9.9 \pm 0.5$ and $T_{K}=22 \pm 1 \mathrm{~K}$. This independent analysis supports the previous one obtained with the data from Fig. 2(b) and interestingly, the adjustable parameter $\alpha$ differs only by $4 \%$ to the corresponding value without coupling to phonons [Fig. 1(c)].

\section{THE SATELLITE PEAKS}

In what follows we focus on the analysis of the evolution with temperature of the two main satellite conductance peaks shown in Fig. 2(a). In contrast to the left satellite, the maximum of the right one is slightly renormalized to higher voltages within the NCA. This might be due to the fact that the NCA does not incorporate renormalization of the bare phonon propagator, and it contributes in a different way for positive and negative frequencies [33]. However, we have verified that both side peaks follow identical temperature dependence [54].

In Fig. 3(a), the satellite conductance at bias voltages near $e V \sim \frac{\hbar}{e} \Omega$ is displayed after removing a linear offset for several

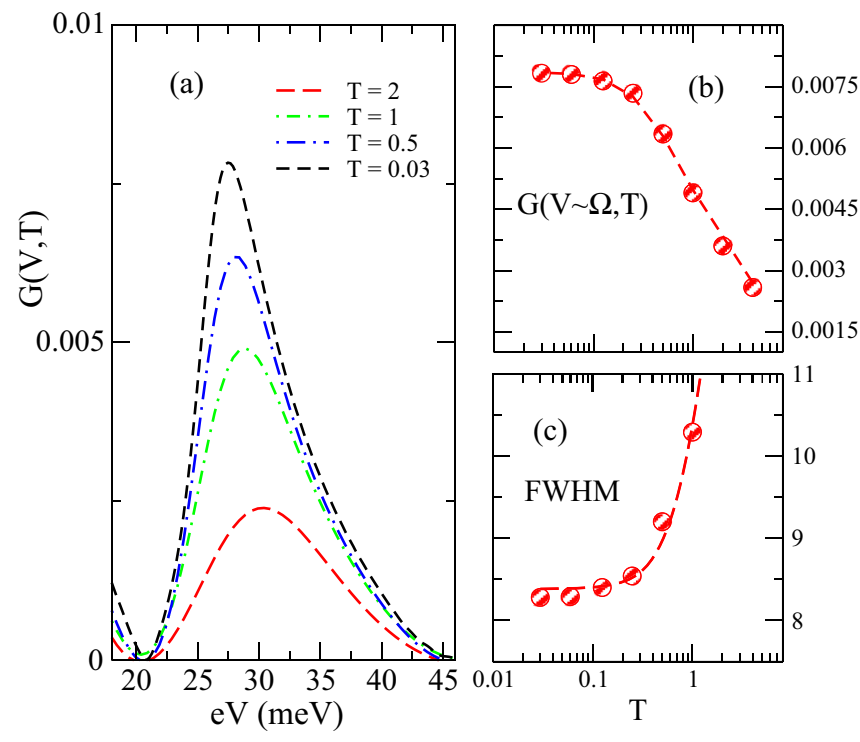

FIG. 3. (a) Differential conductance as a function of bias voltage for several temperatures. (b) Differential conductance $G\left(V \sim \frac{\hbar}{e} \Omega, T\right)$ as a function of temperature (squares) and the corresponding scaling using Eq. (1) (dashed line). (c) FWHM (in meV) of the satellite peak at $V \sim \frac{\hbar}{e} \Omega$ as a function of temperature (squares) and the corresponding scaling (dashed line).

temperatures [55]. From the maximum values, in Fig. 3(b) we built the curve of $G\left(V \sim \frac{\hbar}{e} \Omega, T\right)$ as a function of temperature and its fitting by using Eq. (1). As in the experiment of Rakhmilevitch et al., the values of $G\left(V \sim \frac{\hbar}{e} \Omega, T\right)$ are very well represented by the empirical law, being the adjustable parameter $T_{K}=21 \pm 1 \mathrm{~K}$ in perfect agreement with the Kondo temperature extracted from the central Kondo peak (20 K) [56]. Regarding the left satellite peak, we found $T_{K}=24 \pm 1 \mathrm{~K}$. On the other hand, we find that contrary to what happens for the Kondo peak, the width of the satellite peaks is not related to the Kondo temperature. Figure 3(c) shows the FWHM of the peak at $V \sim \hbar \Omega / e$ as a function of temperature. The parameters extracted from the fit differ with respect to those from the central peak, being $\alpha=6.1 \pm 0.5$ and a low-temperature width $49 \pm 5 \mathrm{~K}$. The increase of the width is expected from the occurrence of inelastic processes. A similar behavior is found for the first satellite peak below the Fermi energy. We could not confirm that the same behavior is valid for further satellite peaks due to the lower intensity of the latter and technical limitations of the NCA calculations.

Since the maxima of the conductance of the satellite peaks have the same temperature dependence as the Kondo peak, and are scaled with almost the same Kondo temperature, we conclude that the side peaks are (broadened) replicas of the Kondo peak. As we stated in the introduction, this is not expected a priori and it would be desirable to have a physical explanation for this. We have extended the variational approach of the Anderson model for the impurity spectral density below the Fermi level explained in Ref. [1] to include phonons. Performing perturbations at lowest order in the electron-phonon interaction $\lambda$, we find a replica at the expected position with relative intensity $(\lambda / \hbar \Omega)^{2}$. Basically, annihilation of the dot electron in the perturbed ground state 


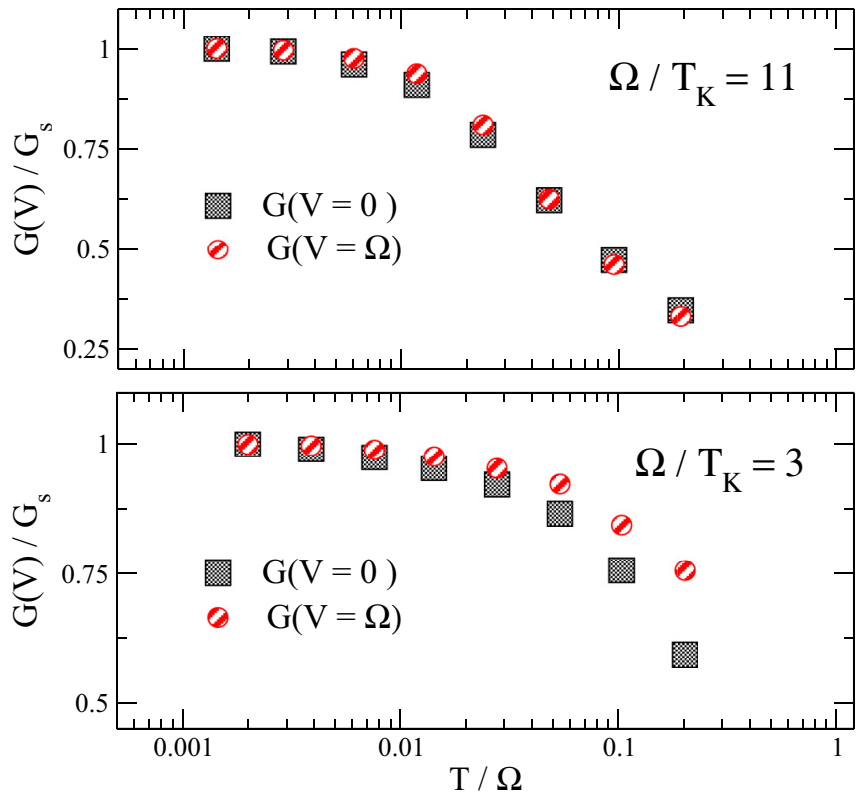

FIG. 4. Top panel: Comparison of the normalized differential conductance, $G(V=0) / G_{s}$ and $G(V \sim \hbar \Omega / e) / G_{s}$, as a function of $k_{B} T / \hbar \Omega$ for the data in Figs. 2 and 3, respectively. Lower panel: same comparison as in the top panel for $\hbar \Omega=5 \mathrm{meV}$, $\Gamma_{R}+\Gamma_{L}=80 \mathrm{meV}, E_{d}=-40 \mathrm{meV}, \lambda=12 \mathrm{meV}$. The adjustable parameter from Eq. (1) is $19 \pm 1 \mathrm{~K}$ in the case of $G(V=0) / G_{s}$ and $30 \pm 1 \mathrm{~K}$ for $G(V \sim \hbar \Omega / e) / G_{s}$.

leads in part (with an amplitude $\lambda / \hbar \Omega$ ) to the same excited states as in the ordinary Anderson model except for the fact that they contain a phonon and thus their energy is shifted by $\hbar \Omega$ (in leading order). This simple approach works qualitatively in the regime $\hbar \Omega \gg k_{B} T_{K}$. However for $\hbar \Omega \approx k_{B} T_{K}$, the energy denominators in a more refined perturbative treatment might be near $\hbar \Omega \pm T_{K}$ and depending on the particular energy of the perturbed Kondo states, the amplitudes might be different, distorting the side peaks.

In order to test the above physical picture, we study a different regime in which $\hbar \Omega$ is of the order of $k_{B} T_{K}$. For simplicity, we use a reduced value of the vibration frequency $\hbar \Omega=5 \mathrm{meV}$, changing the other parameters in order to analyze different values of the ratio $\hbar \Omega / k_{B} T_{K}$. The top panel of Fig. 4 displays a comparison of the normalized differential conductance, $G(V=0) / G_{s}$ and $G(V \sim \hbar \Omega / e) / G_{s}$, as a function of $k_{B} T / \hbar \Omega$ for the data in Figs. 2 and 3, respectively. As we already discussed, in this particular regime for which $\hbar \Omega / k_{B} T_{K}=11$, the evolution with temperature of the zero- bias peak and the satellite conductance peaks is the same. On the other hand, the lower panel shows that the temperature evolution is no longer the same in the case of $\hbar \Omega / k_{B} T_{K}=3$. Here we have used $\hbar \Omega=5 \mathrm{meV}, \Gamma_{R}+\Gamma_{L}=80 \mathrm{meV}, E_{d}=$ $-40 \mathrm{meV}$, and $\lambda=12 \mathrm{meV}$. For this choice of parameters the energy scale obtained from Eq. (1) is found to be $19 \pm 1 \mathrm{~K}$ in the case of $G(V=0) / G_{s}$ and it still represents the Kondo temperature. In fact, this value agrees with the corresponding one obtained from the FWHM of the zero bias anomaly. However, the adjustable parameter extracted from a fitting of the temperature dependence of the inelastic peak at positive voltage $G(V \sim \hbar \Omega / e) / G_{s}$ is found to be $30 \pm 1 \mathrm{~K}$, near twice larger than $T_{K}$. We have verified that this deviation is always present when $k_{B} T_{K}$ approaches $\hbar \Omega$ varying $\hbar \Omega$ from 5 to $21 \mathrm{meV}$ and also $E_{d}$ from -40 to $-90 \mathrm{meV}$. We cannot reach the range for which $\hbar \Omega / k_{B} T_{K} \sim 1$ due to the fact that larger values of $T_{K}$ drive the system towards a mixed valence regime for which there is no universal behavior of the conductance. A possible way to avoid this limitation would be to use unrealistic small values of the electron-phonon interaction $\lambda$.

\section{SUMMARY}

In conclusion, we have verified theoretically that the empirical expression for the temperature dependence of the conductance given by Eq. (1) still works to extract the Kondo scale in transport measurements through molecules with active phonon modes for temperatures $k_{B} T<\hbar \Omega$. It is also able to reproduce the temperature dependence of the out-of-equilibrium conductance peaks at finite bias voltages of the order of $|V| \sim$ $\hbar \Omega / e$ with the same energy scale when $k_{B} T_{K} \ll \hbar \Omega$. These side peaks are however broader than the central Kondo peak due to inelastic effects. In cases for which $k_{B} T_{K} \sim \hbar \Omega$, while the same expression is able to fit the temperature dependence of of $G(V=0)$ and $G(|V| \sim \hbar \Omega / e)$ the resulting energy scales do not coincide with each other, which is an indication of different functional dependence. As a concluding remark, we want to stress here that the ratio $\hbar \Omega / k_{B} T_{K}$ could be very different depending on the experimental system due to the particular frequencies of the vibration modes of the tested molecule and also due to the couplings of the molecule to the metallic contacts.

\section{ACKNOWLEDGMENTS}

We are partially supported by CONICET, Argentina. This work was sponsored by PICT 2013-1045 of the ANPCyT, Argentina, PIP 112-201101-00832 of CONICET, Argentina.
[1] A. C. Hewson, The Kondo Problem to Heavy Fermions (Cambridge University Press, Cambridge, England, 1997).

[2] J. Kondo, Resistance Minimum in Dilute Magnetic Alloys, Prog. Theor. Phys. 32, 37 (1964).

[3] D. Goldhaber-Gordon, H. Shtrikman, D. Mahalu, D. AbuschMagder, U. Meirav, and M. A. Kastner, Kondo effect in a singleelectron transistor, Nature (London) 391, 156 (1998).
[4] S. M. Cronenwett, T. H. Oosterkamp, and L. P. Kouwenhoven, A Tunable Kondo Effect in Quantum Dots, Science 281, 540 (1998).

[5] D. Goldhaber-Gordon, J. Göres, M. A. Kastner, H. Shtrikman, D. Mahalu, and U. Meirav, From the Kondo Regime to the Mixed-Valence Regime in a Single-Electron Transistor, Phys. Rev. Lett. 81, 5225 (1998). 
[6] W. G. van der Wiel, S. de Franceschi, T. Fujisawa, J. M. Elzerman, S. Tarucha, and L. P. Kowenhoven, The Kondo Effect in the Unitary Limit, Science 289, 2105 (2000).

[7] M. Grobis, I. G. Rau, R. M. Potok, H. Shtrikman, and D. Goldhaber-Gordon, Universal Scaling in Nonequilibrium Transport through a Single Channel Kondo Dot, Phys. Rev. Lett. 100, 246601 (2008).

[8] A. V. Kretinin, H. Shtrikman, D. Goldhaber-Gordon, M. Hanl, A. Weichselbaum, J. von Delft, T. Costi, and D. Mahalu, Spin- $\frac{1}{2}$ Kondo effect in an InAs nanowire quantum dot: Unitary limit, conductance scaling, and Zeeman splitting, Phys. Rev. B 84, 245316 (2011).

[9] S. Amasha, A. J. Keller, I. G. Rau, A. Carmi, J. A. Katine, H. Shtrikman, Y. Oreg, and D. Goldhaber-Gordon, Pseudospin-Resolved Transport Spectroscopy of the Kondo Effect in a Double Quantum Dot, Phys. Rev. Lett. 110, 046604 (2013).

[10] W. Liang, M. P. Shores, M. Bockrath, J. R. Long, and H. Park, Kondo resonance in a single-molecule transistor, Nature (London) 417, 725 (2002).

[11] S. Kubatkin, A. Danilov, M. Hjort, J. Cornil, J. L. Brédas, N. Stuhr-Hansen, P. Hedegård, and Th. Bjørnholm, Single-electron transistor of a single organic molecule with access to several redox states, Nature (London) 425, 699 (2003).

[12] L. H. Yu, Z. K. Keane, J. W. Ciszek, L. Cheng, J. M. Tour, T. Baruah, M. R. Pederson, and D. Natelson, Kondo Resonances and Anomalous Gate Dependence in the Electrical Conductivity of Single-Molecule Transistors, Phys. Rev. Lett. 95, 256803 (2005).

[13] M. N. Leuenberger and E. R. Mucciolo, Berry-Phase Oscillations of the Kondo Effect in Single-Molecule Magnets, Phys. Rev. Lett. 97, 126601 (2006).

[14] J. J. Parks, A. R. Champagne, G. R. Hutchison, S. Flores-Torres, H. D. Abruña, and D. C. Ralph, Tuning the Kondo Effect with a Mechanically Controllable Break Junction, Phys. Rev. Lett. 99, 026601 (2007).

[15] N. Roch, S. Florens, V. Bouchiat, W. Wernsdorfer, and F. Balestro, Quantum phase transition in a single-molecule quantum dot, Nature (London) 453, 633 (2008).

[16] G. D. Scott, Z. K. Keane, J. W. Ciszek, J. M. Tour, and D. Natelson, Universal scaling of nonequilibrium transport in the Kondo regime of single molecule devices, Phys. Rev. B 79, 165413 (2009).

[17] J. J. Parks, A. R. Champagne, T. A. Costi, W. W. Shum, A. N. Pasupathy, E. Neuscamman, S. Flores-Torres, P. S. Cornaglia, A. A. Aligia, C. A. Balseiro, G. K.-L. Chan, H. D. Abruñ a, and D. C. Ralph, Mechanical Control of Spin States in Spin-1 Molecules and the Underscreened Kondo Effect, Science 328, 1370 (2010).

[18] S. Florens, A. Freyn, N. Roch, W. Wernsdorfer, F. Balestro, P. Roura-Bas, and A. A. Aligia, Universal transport signatures in two-electron molecular quantum dots: gate-tunable Hund's rule, underscreened Kondo effect and quantum phase transitions, J. Phys. Condens. Matter 23, 243202 (2011); references therein.

[19] R. Vincent, S. Klyatskaya, M. Ruben, W. Wernsdorfer, and F. Balestro, Electronic read-out of a single nuclear spin using a molecular spin transistor, Nature (London) 488, 357 (2012).

[20] R. M. Potok, I. G. Rau, Hadas Shtrikman, Yuval Oreg and D. Goldhaber-Gordon, Observation of the two-channel Kondo effect, Nature (London) 446, 167 (2007).
[21] A. J. Keller, S. Amasha, I. Weymann, C. P. Moca, I. G. Rau, J. A. Katine, Hadas Shtrikman, G. Zarànd, and D. Goldhaber-Gordon, Emergent SU(4) Kondo physics in a spincharge-entangled double quantum dot, Nat. Phys. 10, 145 (2014).

[22] P. S. Cornaglia, P. Roura Bas, A. A. Aligia and C. A. Balseiro, Quantum transport through a stretched spin-1 molecule, Europhys. Lett. 93, 47005 (2011).

[23] R. H. M. Smit, Y. Noat, C. Untiedt, N. D. Lang, M. C. van Hemert, and J. M. van Ruitenbeek, Measurement of the conductance of a hydrogen molecule, Nature (London) 419, 906 (2002)

[24] A. N. Pasupathy, J. Park, C. Chang, A. V. Soldatov, S. Lebedkin, R. C. Bialczak, J. E. Grose, L. A. K. Donev, J. P. Sethna, D. C. Ralph, and P. L. McEuen, Vibration-Assisted Electron Tunneling in $\mathrm{C}_{140}$ Transistors, Nano. Lett. 5, 203 (2005).

[25] H. Park, J. Park, A. K. L. Lim, E. H. Anderson, A. P. Alivisatos, and P. L. McEuen, Nanomechanical oscillations in a single-C60 transistor, Nature (London) 407, 57 (2000).

[26] N. B. Zhitenev, H. Meng, and Z. Bao, Conductance of Small Molecular Junctions, Phys. Rev. Lett. 88, 226801 (2002).

[27] L. H. Yu, Z. K. Keane, J. W. Ciszek, L. Cheng, M. P. Stewart, J. M. Tour, and D. Natelson, Inelastic Electron Tunneling via Molecular Vibrations in Single-Molecule Transistors, Phys. Rev. Lett. 93, 266802 (2004).

[28] I. Fernández-Torrente, K. J. Franke, and J. I. Pascual, Vibrational Kondo Effect in Pure Organic Charge-Transfer Assemblies, Phys. Rev. Lett. 101, 217203 (2008).

[29] D. Rakhmilevitch, R. Korytár, A. Bagrets, F. Evers, and O. Tal, Electron-Vibration Interaction in the Presence of a Switchable Kondo Resonance Realized in a Molecular Junction, Phys. Rev. Lett. 113, 236603 (2014).

[30] A. Mugarza, C. Krull, R. Robles, S. Stepanow, G. Ceballos, and P. Gambardella, Spin coupling and relaxation inside moleculemetal contacts, Nat. Commun. 2, 490 (2011).

[31] T. A. Costi, Kondo Effect in a Magnetic Field and the Magnetoresistivity of Kondo Alloys, Phys. Rev. Lett. 85, 1504 (2000).

[32] J. Paaske and K. Flensberg, Vibrational Sidebands and the Kondo Effect in Molecular Transistors, Phys. Rev. Lett. 94, 176801 (2005).

[33] P. Roura-Bas, L. Tosi, and A. A. Aligia, Nonequilibrium transport through magnetic vibrating molecules, Phys. Rev. B 87, 195136, (2013). references therein.

[34] A. C. Hewson and D. Meyer, Numerical Renormalization Group Study of the Anderson-Holstein Impurity Model, J. Phys. Condens. Matter 14, 427 (2002).

[35] L. Arrachea and M. J. Rozenberg, Quantum Monte Carlo method for models of molecular nanodevices, Phys. Rev. B 72 , 041301(R) (2005).

[36] P. S. Cornaglia, Gonzalo Usaj, and C. A. Balseiro, Electronic transport through magnetic molecules with soft vibrating modes, Phys. Rev. B 76, 241403(R) (2007).

[37] R. Žitko and Th. Pruschke, Energy resolution and discretization artifacts in the numerical renormalization group, Phys. Rev. B 79, 085106 (2009).

[38] R. C. Monreal and A. Martin-Rodero, Equation of motion approach to the Anderson-Holstein Hamiltonian, Phys. Rev. B 79, 115140 (2009). 
[39] N. S. Wingreen and Y. Meir, Anderson model out of equilibrium: Noncrossing-approximation approach to transport through a quantum dot, Phys. Rev. B 49, 11040 (1994).

[40] P. Roura Bas and A. A. Aligia, Nonequilibrium transport through a singlet-triplet Anderson impurity, Phys. Rev. B 80, 035308 (2009).

[41] L. Tosi, P. Roura-Bas, and A. A. Aligia, Orbital Kondo spectroscopy in a double quantum dot system, Phys. Rev. B 88, 235427, (2013).

[42] L. Tosi, P. Roura-Bas, and A. A. Aligia, Restoring the SU(4) Kondo regime in a double quantum dot system, J. Phys. Condens. Matter 27, 335601 (2015).

[43] S. Di Napoli, A. Weichselbaum, P. Roura-Bas, and A. A. Aligia, Non-Fermi liquid behavior in nonequilibrium transport through Co doped Au chains connected to four-fold symmetric leads, Phys. Rev. B 90, 125149 (2014).

[44] P. Roura Bas and A. A. Aligia, Nonequilibrium dynamics of a singlet triplet-Anderson impurity near the quantum phase transition, J. Phys.: Condens. Matter 22, 025602 (2010).

[45] P. Roura-Bas, Universal scaling in transport out of equilibrium through a single quantum dot using the noncrossing approximation, Phys. Rev. B 81, 155327 (2010).

[46] While the NCA cannot reach $T=0$, the conductance has already reached its saturation value for temperatures $\sim 0.1 T_{K}$ for which the NCA is still reliable.

[47] Y. Meir and N. S. Wingreen, Phys. Rev. Lett. 68, 2512 (1992).

[48] A. A. Aligia, P. Roura-Bas, and S. Florens, Impact of capacitance and tunneling asymmetries on Coulomb blockade edges and
Kondo peaks in nonequilibrium transport through molecular quantum dots, Phys. Rev. B 92, 035404 (2015).

[49] N. Tsukahara, S. Shiraki, S. Itou, N. Ohta, N. Takagi, and M. Kawai, Evolution of Kondo Resonance from a Single Impurity Molecule to the Two-Dimensional Lattice, Phys. Rev. Lett. 106, 187201 (2011).

[50] Other forms of this kind of expression, like $\frac{e^{2}}{8 k_{B}^{2}}(\mathrm{FWHM})^{2}=$ $(\pi T)^{2} / 2+T_{K}^{2}$, are also found in the literature $[29,12]$.

[51] L. Tosi, P. Roura Bas, A. M. Llois, and A. A. Aligia, Out of equilibrium Anderson model: Conductance and Kondo temperature, Physica B 407, 3263 (2012).

[52] A. A. Aligia, Nonequilibrium self-energies, $\mathrm{Ng}$ approach, and heat current of a nanodevice for small bias voltage and temperature, Phys. Rev. B 89, 125405 (2014); references therein.

[53] I. J. Hamad, C. Gazza, J. A. Andrade, A. A. Aligia, P. S. Cornaglia, and P. Roura Bas, Scaling of conductance through quantum dots with magnetic field, Phys. Rev. B 92, 195113 (2015); references therein.

[54] We have also replaced the bare phonon propagator by the dressed one including corrections to second order in $\lambda$, resulting in very small changes in the satellite peaks and to the same temperature dependence.

[55] The satellite peaks are mounted on the tail of the main Kondo peak (see Fig. 1). We have subtracted this tail assuming a linear dependence, which is justified due ti the small curvature of the main peak at the energies of the satellites.

[56] We note that if instead of following the maximum of the satellite peak in $G(V)$ for each temperature, a fixed $V$ is taken (for example the position of the maximum at $T=0$ ), the scaling law is not obeyed. 